\title{
Trunk Flexibility Improvement in Response to Powered Assisted Exercise
}

\author{
B.S. Bains \\ School of Physiotherapy, Aihmsa College, Kuala Lumpur, Malaysia \\ E-mail: datobains@gmail.com \\ Gurujit Kaur (Corresponding author) \\ School of Physiotherapy, Aihmsa College, Kuala Lumpur, Malaysia \\ E-mail:jit_88@hotmail.com \\ Hassan Sadeghi \\ School of Physiotherapy, Aihmsa College Kuala Lumpur, Malaysia \\ E-mail: Hassan.sadeghi81@yahoo.com \\ AmeerudinGulam Husain \\ BainsPhysio Group Practice, JalanMelawati, Kuala Lumpur, Malaysia \\ E-mail:bainsphysio@gmail.com \\ Karminder Singh \\ BainsPhysio Group Practice, JalanMelawati, Kuala Lumpur, Malaysia \\ E-mail:physiofitness@ymail.com
}

Received: 04-06- 2016

Accepted: 16-07- 2016

Published: 31-07- 2016

doi:10.7575/aiac.ijkss.v.4n.3p.50

URL: http://dx.doi.org/10.7575/aiac.ijkss.v.4n.3p.50

\begin{abstract}
Background study: Flexibility in human spine has always plays an important role in dexterity and seamless ambulatory activities. When optimum range is not maintained by the trunk column, due to lack of flexibility, the posture gets affected resulting in reduce trunk rotation flexibility and mobility hence loss of complete trunk rotation. Objective: The purpose of this study is to determine the effect of Shapemaster Power Assisted Exercise Equipment (SPAEE) on trunk flexibility. Methodology: Twenty healthy individual ages between 40 to 60 years were randomly divided into control and exercise groups. Shapemaster exercise program performed two times per week for 5 weeks and 45 minutes per session. Before and after 10 sessions of Shapemaster exercise protocol, Seated trunk rotation test was used to measure trunk flexibility. Results: Repeated measurement ANOVA were used to analysis data between groups. The results of this study illustrated that after $10^{\text {th }}$ sessions trunk flexibility significantly improved $(\mathrm{F}(1.0,18)=11.732, \mathrm{p}<$ 0.003). Conclusion: In conclusion results were determined that SPAEE is safe and it did effectively enhance flexibility among individual healthy adults.

Keyword: Shapemaster Power Assisted Exercise Equipment (SPAEE), Trunk Flexibility, Healthy individual 1. Introduction

Flexibility is the body's ability to move freely around its joints within range of movements depending on tissues make up (genetic) activity (Stathokostas, McDonald, Little, \& Paterson, 2013). Flexibility is also important to help prevent injuries and assist in recovery to improve performance (Montero-Fernandez \& Serra-Rexach, 2013; Purepong, Jitvimonrat, Boonyong, Thaveeratitham, \& Pensri, 2012). Back flexibility helps to reduce fatigue and improved body position and further helps to determine the position of the body parts in relaxation, in relation to the other body segments at a given time (Nagai et al., 2014). Flexibility can effect daily activities, athletes performance (Johnson, Kim, Yu, Saliba, \& Grindstaff, 2012), and companies worker (Shariat, Bahri Mohd Tamrin, Daneshjoo, \& Sadeghi, 2015). The Lumbar (lower back) lordotic curve and thoracic (mid back), this bicurve spine plays an important role to stand upright most of the time and have dexterities on our upper extremities (Claus, Hides, Moseley, \& Hodges, 2016) hence trunk flexibility and mobility prevent low back pain and enhance body posture (Liddle, Baxter, \& Gracey, 2004). In today society many people tend to lose that bicurve spine length of the trunk in sitting and standing when adopting a slouch posture, resulting in a loss of complete trunk rotation flexibility and mobility whereby it leads to thoracic stiffness. A considerable amount of literature has been published on trunk flexibility. A recent study of (Nagai et al., 2015) reported the relationship between lumbar spine flexibility and low back pain, and they found that improvement of trunk flexibility and side-to-side symmetries could reduce low back pain. Another study suggested that flexibility may be a predictor of arterial stiffening, independent of other components of fitness (Yamamoto et al., 2009). Regular
\end{abstract}


exercises and structured stretching improved functional mobility and flexibility of older adults. Studies of (Phrompaet, Paungmali, Pirunsan, \& Sitilertpisan, 2011; Sekendiz, Altun, Korkusuz, \& Akın, 2007) confirmed that Pilates significantly improved trunk mobility and flexibility. SPAEE is a new innovation, whereby they are fitted with motors and gearboxes that move the arm handles or foot pedals through predetermined speed and range of motion. Today SPAEE are recognized equipment rediscovered and refined to forms an alternative way to exercise rather than that with high impact, percussive and joint stressing routines that are found in the traditional gym environment. The advantage of using SPAEE is that it forms an integrated part of isotonic, isokinetic, passive and cardio vascular exercise training system. One of the SPAEE is called "Fly's and Thighs"; It helps to exercise and stretch all the major muscle groups in the body, whilst the soothing motion promotes relaxation (Jacobson, Smith, Fronterhouse, Kline, \& Boolani, 2012). Originally SPAEE were designed to help people who suffer from poliomyelitis; an infectious viral disease that affects the central nervous system and that could cause temporary or permanent paralysis such as muscle wastage due immobility. It was soon recognized, that, muscle tone is greatly improved and further improves circulation, strength and agility. Jacobson et al., (2012) demonstrated that SPAEE when used actively, improved balance, both upper and lower body, muscle endurance in elderly participants in a safe exercise environment. Nevertheless, there has been no previous studies conducted on assessing the effectiveness of SPAEE on the trunk flexibility; the purpose of this study was to determine the effectiveness of 10 sessions of SPAEE on trunk flexibility of healthy individual adults. It has been hypothesized that in 5 weeks SPAEE exercises improved trunk flexibility among healthy individuals.

\section{Methodology}

\subsection{Participants}

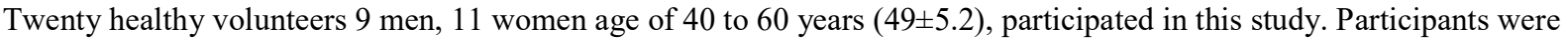
selected from BainsPhysio ${ }^{\circledR}$ (A Physiotherapy Clinic) at Taman Melawati, Kuala Lumpur, Malaysia serving the public in the surrounding community area coming for various musculoskeletal and other conditions needing physiotherapy. All participants signed a consent form before starting the study and were screened for eligibility by the senior physiotherapists. Exclusion criteria taken into account were with pathological condition affecting spine, rib, shoulder, hip, or knee within the past 6 months with history of scoliosis or the presence of spinal internal fixation, a rheumatologic, prolapsed intervertebral disc, vertigo, lower back and upper neck pain. After baseline assessment, eligible participants randomly divided into control and experimental groups. The study was conducted according to the principles of the Declaration of Helsinki (Aragon, 2010; Kargarfard et al., 2015).

2.2 Test procedure

Seated trunk rotation test was used to measure the trunk flexibility in right and left side (Aragon, Oyama, Oliaro, Padua, \& Myers, 2012; Johnson \& Grindstaff, 2010). The subject was asked to sit on a chair with their feet together and flat on the ground, with the trunk in upright posture and arms across their chest. The subject was asked to rotate to the right as far as possible with no discomfort. A goniometer was used to measure the amount of rotation. The test was repeated the same on the left with subject rotating as far as possible. The intra-class correlation coefficient ( ICC) and standard error of measurement ( SEM) for the right side was $\operatorname{ICC}(2, \mathrm{k})=.798, \mathrm{SEM}=4.1$ degree and for the left side was $\operatorname{ICC}(2, \mathrm{k})=.727 / \mathrm{SEM}=5.0$ degree (Aragon, 2010).

2.3 Exercise protocol

Experimental group attended 10 sessions of exercise protocol two times per week and 45 minutes per session. They were assisted and monitored by two physiotherapists for each session of exercise. To avoid risk of injuries participations were taught exercise protocols (Sadeghi, Shariat, Asadmanesh, \& Mosavat, 2013). Before each session they were given ten (10) minutes of general walking for warming up on treadmill and gentle active stretching such as triceps stretch, bicep stretch, rolling of shoulder forward and backward, bending forward stretch in standing and breathing exercises (inhalation and exhalation) to bring the body to a condition at which it safely responds to nerve signals for quick and efficient action. After warm up sessions, participants were assisted towards SPAEE (Fly's and thighs) and instructions were given about position on this equipment in the supine lying (Figure 1). Alignment of head neck shoulder is strictly watched to avoid any indirect injuries or strain to the neck or lower back. Interlocking both hands at SPAEE arm handle on (One side), left side is essential to monitor the console while performing trunk rotation to allow participant to monitor timing.

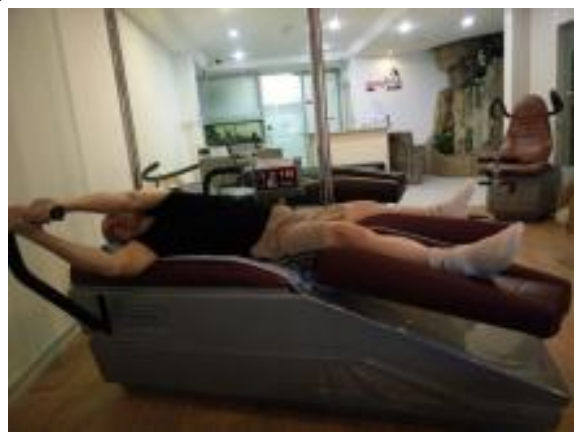

Figure 1. SPAEE - Fly's \& Thighs 


\subsection{Data Analysis}

Data were analyzed using SPSS version 22. Two ways repeated measurements ANOVA were used to analyze pre and post data for both experimental and control groups. The level of significant was set at $\mathrm{p}<0.05$. Normality of data was checked using the Shapiro-Wilk test.

3. Results

Characteristics of participants such as age, weight and height were shown in Table 1

Table 1. characteristic of participants

\begin{tabular}{lcc}
\hline Variable & $\mathrm{N}$ & Mean \pm SD \\
\hline Age & 20 & $49.85 \pm 5.2$ \\
Weight & 20 & $65.30 \pm 5.2$ \\
Height & 20 & $165.75 \pm 5.1$ \\
\hline
\end{tabular}

The repeated measures ANOVA shows significant differences between pre- and post-tests $\left(\mathrm{F}_{(1.0,18}\right)=128.207, p<$ $0.001)$ and the results show a significant interaction between time and groups $\left.\left(\mathrm{F}_{(1.0,18}\right)=196.12, p<0.001\right)$, and there were significant differences between control and experimental group $\left.\left(\mathrm{F}_{(1.0,18}\right)=11.732, p<0.003\right)$. There was no significant difference between flexibility of left and right side trunk rotation $\left.\left(\mathrm{F}_{(1.0,18.0}\right)=.267, p<0.611\right)$. Table 2 shows the mean differences between experimental and control group in pre-test and post-test, and also side flexibility of participants.

Table 2. Mean differences between experimental and control group in pre and post-test

\begin{tabular}{lccccc}
\hline & \multicolumn{2}{c}{ Measure } & Mean & S E & P Value \\
\hline Group & Control & Exercise & $1.53^{*}$ & .445 & 0.003 \\
Time & Pre test & Post Test & $2.43^{*}$ & .214 & 0.000 \\
\multirow{2}{*}{ Side Flexibility } & Left Side & Right side & .075 & .145 & 0.611 \\
\hline
\end{tabular}

\section{Discussion}

This study conducted to design the effectiveness of SPAEE on trunk flexibility of individual adults. Results of this study illustrated that 10 sessions of SPAEE exercise significantly improved trunk flexibility. SPAEE improved muscular endurance and functional capacity among elderly participants (Johnson et al., 2012). They reported that SPAEE exercise is safe and useful for upper and lower body muscle endurance. The results of this study are in line with previous study of (Sekendiz et al., 2007) who conducted that Pilates exercise improved flexibility of elder women. By stretching of joint range of motion during Pilates movement, muscle was stretch. In contrast, exercises machine are safer than conventional gym exercise, because these machine guide and control the path of exercise such as load, intensity and range of motion (Kerr, Collins, \& Comstock, 2010), but in conventional gym exercises, the participants require to maintain and control the body to do the particular movements which may contribute to injuries among untrained participants. Therefore, SPAEE is recommended to be used for any patients because there is no need to stabilize and control posture and it thus reduces difficulties and limitation in rehabilitations program. There are several possible explanations for this result, firstly, SPAEE keeps the joints movement well in control and due to that the speed can be accelerated safely, and this mimics isokinetic exercises. As has been suggested that confidence in the safety of the exercise affects adherence and participants effort to maintaining exercise for a prolong period (Brassington, Atienza, Perczek, DiLorenzo, \& King, 2002). Secondly, in this study is found that there is no dropped-out participants due to delayed onset of muscle soreness or injuries during exercise. Thirdly, the results of the current study illustrated that, flexibility of both right and left side is improved. Since the pattern of exercise is symmetrical on both sides, no significant difference was seen between right and left side of the participant in terms of strength and tone. These results explain that the use of SPAEE improved range of motion bilaterally with equally stretches on both side. On the contrary this may not happen with traditional flexibility exercise because in traditional flexibility exercise participants may not get the accurate range of motion and may not keep with the consistent intensity of motion. Finally a number of important limitations needed to be considered. Firstly the numbers of participant in this study is small and are limited to healthy participants. Second, there was no traditional exercise control group in this study. Therefore, further research can be investigated in patients with musculoskeletal disorders and it would be interesting to compare SPAEE with other types of exercises. 


\section{Conclusion}

In conclusion, this study has shown that SPAEE have improved the right and left side of the trunk flexibility among healthy individual. SPAEE can control the range of motion, load, and intensity of exercises. It is also recognized as a safe exercise method as compared to the traditional exercise for fitness and rehabilitation forms.

\section{References}

Aragon, V. J. (2010). A comparison of trunk rotation flexibility and trunk rotation kinematics during throwing between division I collegiate softball position players with and without a history of shoulder or elbow pain. (Masters), University of North Carolina.

Aragon, V. J., Oyama, S., Oliaro, S. M., Padua, D. A., \& Myers, J. B. (2012). Trunk-rotation flexibility in collegiate softball players with or without a history of shoulder or elbow injury. Journal of athletic training, 47(5), 507.

Brassington, G. S., Atienza, A. A., Perczek, R. E., DiLorenzo, T. M., \& King, A. C. (2002). Intervention-related cognitive versus social mediators of exercise adherence in the elderly. American journal of preventive medicine, 23(2), 80-86.

Claus, A. P., Hides, J. A., Moseley, G. L., \& Hodges, P. W. (2016). Thoracic and lumbar posture behaviour in sitting tasks and standing: Progressing the biomechanics from observations to measurements. Applied ergonomics, 53, 161168.

Jacobson, B. H., Smith, D., Fronterhouse, J., Kline, C., \& Boolani, A. (2012). Assessment of the benefit of powered exercises for muscular endurance and functional capacity in elderly participants. Journal of Physical Activity and Health, 9(7), 1030.

Johnson, K. D., \& Grindstaff, T. L. (2010). Thoracic rotation measurement techniques: clinical commentary. North American Journal of Sports Physical Therapy, 5(4), 252-256.

Johnson, K. D., Kim, K.-M., Yu, B.-K., Saliba, S. A., \& Grindstaff, T. L. (2012). Reliability of thoracic spine rotation range-of-motion measurements in healthy adults. Journal of athletic training, 47(1), 52-60.

Kargarfard, M., Shariat, A., Shaw, B. S., Shaw, I., Lam, E. T., Kheiri, A., . . Tamrin, S. B. (2015). Effects of polluted air on cardiovascular and hematological parameters after progressive maximal aerobic exercise. Lung, 193(2), $275-281$.

Kerr, Z. Y., Collins, C. L., \& Comstock, R. D. (2010). Epidemiology of weight training-related injuries presenting to United States emergency departments, 1990 to 2007. The American Journal of Sports Medicine, 38(4), 765-771.

Liddle, S. D., Baxter, G. D., \& Gracey, J. H. (2004). Exercise and chronic low back pain: what works? Pain, 107(1), $176-190$

Montero-Fernandez, N., \& Serra-Rexach, J. (2013). Role of exercise on sarcopenia in the elderly. European journal of physical and rehabilitation medicine, 49(1), 131-143.

Nagai, T., Abt, J. P., Sell, T. C., Clark, N. C., Smalley, B. W., Wirt, M. D., \& Lephart, S. M. (2014). Neck proprioception, strength, flexibility, and posture in pilots with and without neck pain history. Aviation, space, and environmental medicine, 85(5), 529-535.

Nagai, T., Abt, J. P., Sell, T. C., Keenan, K. A., Clark, N. C., Smalley, B. W., . . Lephart, S. M. (2015). Lumbar spine and hip flexibility and trunk strength in helicopter pilots with and without low back pain history. Work, 52(3), 715-722.

Phrompaet, S., Paungmali, A., Pirunsan, U., \& Sitilertpisan, P. (2011). Effects of pilates training on lumbo-pelvic stability and flexibility. Asian Journal of sports medicine, 2(1), 16-22.

Purepong, N., Jitvimonrat, A., Boonyong, S., Thaveeratitham, P., \& Pensri, P. (2012). Effect of flexibility exercise on lumbar angle: a study among non-specific low back pain patients. Journal of bodywork and movement therapies, 16(2), 236-243.

Sadeghi, H., Shariat, A., Asadmanesh, E., \& Mosavat, M. (2013). The Effects of core stability Exercise on the dynamic balance of volleyball players. International Journal of Applied Exercise Physiology, 2(2), 1-10.

Sekendiz, B., Altun, Ö., Korkusuz, F., \& Akın, S. (2007). Effects of Pilates exercise on trunk strength, endurance and flexibility in sedentary adult females. Journal of bodywork and movement therapies, 11(4), 318-326.

Shariat, A., Bahri Mohd Tamrin, S., Daneshjoo, A., \& Sadeghi, H. (2015). The Adverse Health Effects of Shift Work in Relation to Risk of Illness/Disease: A Review. Acta Medica Bulgarica, 42(1), 63-72.

Stathokostas, L., McDonald, M. W., Little, R., \& Paterson, D. H. (2013). Flexibility of older adults aged 55-86 years and the influence of physical activity. Journal of aging research, 2013.

Yamamoto, K., Kawano, H., Gando, Y., Iemitsu, M., Murakami, H., Sanada, K., Tabata, I. (2009). Poor trunk flexibility is associated with arterial stiffening. American Journal of Physiology-Heart and Circulatory Physiology, 297(4), H1314-H1318. 